\title{
Exemestane-Induced Eosinophilic Colitis in a Patient with Grade 3 Ductal Carcinoma In-Situ: A Case Report and Review of the Lit- erature
}

\author{
Kristyn Sanders ${ }^{1 \#}$, Shannon Lim ${ }^{1 \#}$, Sandra Ortega RN $^{2 \#}$, Mark Gimbe ${ }^{3 \#}$ and Lida Mina ${ }^{4 *^{*}}$ \\ ${ }^{1}$ Department of Pharmacy, Banner MD Anderson Cancer Center, USA \\ ${ }^{2}$ Department of Case Management, Banner MD Anderson Cancer Center, USA \\ ${ }^{3}$ Department of Surgical Oncology, Banner MD Anderson Cancer Center, USA \\ ${ }^{4}$ Department of Breast Medical Oncology, Banner MD Anderson Cancer Center, USA \\ ${ }^{\#}$ Equal contribution: All authors contributed equally to the writing and publication of this manuscript
}

*Corresponding author: Lida Mina, MD, Department of Breast Medical Oncology, Banner MD Anderson Cancer Center, USA

\begin{abstract}
Purpose: A case report of eosinophilic colitis associated with exemestane use is presented.

Summary: Approximately after three months of exemestane therapy for breast cancer risk reduction, a 60-year-old female with estrogen receptor positive, right breast ductal carcinoma in situ (DCIS) presented to the emergency room with severe abdominal pain, nausea, vomiting, and diarrhea. Imaging studies revealed severe colitis of the transverse coIon. No other medications were started except for exemestane that would have contributed to the patient's symptoms. Outpatient workup included stool cultures, laboratory studies, and esophagogastroduodenoscopy and colonoscopy with biopsies. Results of the studies were significant for eosinophilic colitis and moderate to borderline severe colitis with increased eosinophilia. The patient was subsequently started on corticosteroids and discontinued exemestane. Her symptoms resolved in two weeks. Approximately two months later, the patient was started on tamoxifen for breast cancer prevention, which she tolerated well.
\end{abstract}

Conclusion: Exemestane was deemed the probable cause of the acute episode of eosinophilic colitis in this case. One other prior case with aromatase inhibitor-induced eosinophilic colitis has been reported with letrozole, however this is the first case report of eosinophilic colitis associated with exemestane.

\section{Background}

Exemestane is an irreversible, steroidal aromatase in-activator that structurally resembles androstenedione [1]. The pharmacological activity of exemestane is centered on the conversion of the drug to an intermediate that irreversibly blocks the active site of aromatase thereby rendering the enzyme permanently inactivated [1]. The inactivation of aromatase results in lower levels of circulating hormones that can increase the growth and size of hormone receptor positive breast cancers [1].

Exemestane first received FDA approval in the United States in 1999 for the treatment of advanced breast cancer in post-menopausal women whose tumors have stopped responding to tamoxifen [2]. Currently, it is approved for: the adjuvant treatment of postmenopausal women with estrogen receptor positive early stage breast cancer who have received two to three years of tamoxifen (an estrogen receptor antagonist) and are switched to exemestane for completion of a total of five years of adjuvant hormonal therapy $[1,3,4]$. It is also approved for advanced breast cancer in women whose disease has progressed following tamoxifen [1]. The American Society of Clinical Oncology (ASCO) clinical practice guidelines also recommends the use of

Citation: Sanders K, Lim S, Ortega S, Gimbel M, Mina L (2019) Exemestane-Induced Eosinophilic Colitis in a Patient with Grade 3 Ductal Carcinoma In-Situ: A Case Report and Review of the Literature. Int J Cancer Clin Res 6:104. doi.org/10.23937/2378-3419/1410104

Accepted: February 05, 2019; Published: February 07, 2019

Copyright: (c) 2019 Sanders K, et al. This is an open-access article distributed under the terms of the Creative Commons Attribution License, which permits unrestricted use, distribution, and reproduction in any medium, provided the original author and source are credited. 
Table 1: Summarizes all the large trials evaluating the use of exemestane in the adjuvant, metastatic, and preventative settings.

\section{Adjuvant}

Morden JP, et al. [4]

Placido SD, et al. [5]

Pagani O, et al. [8]

Goss PE, et al. [13]

Derks MG, et al. [7]

\section{Metastatic}

Campos SM, et al. [9]

Beresford M, et al. [10]

Baselga J, et al. [11]

\section{Prevention}

Goss PE, et al. [13]
Exemestane after 2 to 3 years of tamoxifen

Anastrozole vs. Exemestane vs. Letrozole upfront or after 2 years of tamoxifen Exemestane plus ovarian suppression vs. Tamoxifen plus ovarian suppression Exemestane vs. Anastrozole

Tamoxifen and Exemestane switch therapy vs. Exemestane alone

Exemestane vs. Anastrozole

Systematic review

Exemestane and Everolimus vs. Exemestane and Placebo

Exemestane vs. Placebo exemestane in the setting of breast cancer prevention $[5,6]$.

In the adjuvant setting, exemestane has been studied in both pre- and post-menopausal women [2,5,79]. It has not been shown to be superior to other aromatase inhibitors such as anastrozole or letrozole in the post-menopausal setting $[2,5,7,8]$. Additionally, five years of aromatase inhibitor treatment was not found to be superior to two years of treatment with tamoxifen followed by three years of aromatase inhibitor therapy [7]. In the pre-menopausal setting, exemestane in combination with ovarian suppression was superior to tamoxifen with ovarian suppression in decreasing disease recurrence [9]. There was no difference in overall survival between the two therapies [9].

In patients with metastatic disease, exemestane has been compared to multiple other aromatase inhibitors [10-12]. When compared with anastrozole in patients with visceral metastases, there was no difference in outcomes [10]. A systematic review evaluating ten different trials that assessed the switch between non-steroidal aromatase inhibitors (letrozole and anastrozole) and exemestane was conducted [11]. Out of ten, one was a randomized control trial, and the other nine were either non-randomized comparative studies or single arm observational studies [11]. Clinical benefit was seen in patients with relapsed disease who switched from non-steroidal aromatase inhibitors to exemestane and from exemestane to a non-steroidal aromatase inhibitor [11]. Additionally, exemestane has been studied in combination with everolimus in patients previously treated with non-steroidal aromatase inhibitors and demonstrated improvement in progression free survival [12].

Exemestane is also used for reducing the risk of invasive breast cancer in post-menopausal women $\geq 35$ years of age with a five-year absolute risk $\geq 1.66 \%$ [6]. There is one randomized placebo-controlled trial that has evaluated the use of exemestane for the prevention of invasive breast cancer [13]. Patients with various breast cancer risk factors including prior atypical ductal or lobular hyperplasia or lobular carcinoma in situ and ductal carcinoma in situ with mastectomy were included [13]. Exemestane was found to reduce invasive breast cancer in postmenopausal women who were found to be at moderately increased risk for breast cancer [13] (Table 1).

Exemestane is overall well tolerated with side effects that are common to other aromatase inhibitors such as hot flashes, bone loss, cholesterol metabolism, and musculoskeletal effects [14]. Exemestane did appear to have a larger effect on bone loss and cholesterol metabolism when compared with other aromatase inhibitors [15]. The most common gastrointestinal side effect observed in clinical trials was nausea; other gastrointestinal side effects reported were diarrhea, heartburn, vomiting, constipation, abdominal pain, and gastritis [1,2,5-13]. While eosinophilic colitis has been observed with letrozole [16], there have not been any reports with exemestane.

Eosinophilic colitis (EC) is a type of eosinophilic gastrointestinal disorder (EGID) characterized by eosinophilic infiltration of the intestinal wall involving the small intestine and less commonly the colon [17]. It is considered to be one of the rarer forms of EGIDs with a twoyear prevalence rate of approximately $0.003 \%$ [17]. Patients often present with non-specific symptoms such as abdominal pain, nausea, loss of appetite, and diarrhea [17]. Additionally, the diagnosis of EC typically depends on the increased number of eosinophils seen on mucosal biopsy [17]. While there are a variety of causes such as parasitic and bacterial infections, inflammatory bowel disease, hypereosinophilic syndrome, connective tissue disorders, and myeloproliferative neoplasms, it can also be caused by hypersensitivity to various pharmacological agents [17]. Common agents associated with EC include carbamazepine, gold compounds, clozapine, rifampicin, clofazamine, gemfibrozil, azathioprine, enalapril, naproxen, interferon, and tacrolimus [17-26].

There are currently no randomized controlled trials evaluating the various treatment modalities for EC. Treatment is typically dependent upon the severity of clinical manifestations and includes dietary elimination, steroids, montelukast, cromolyn, ketotifen, 


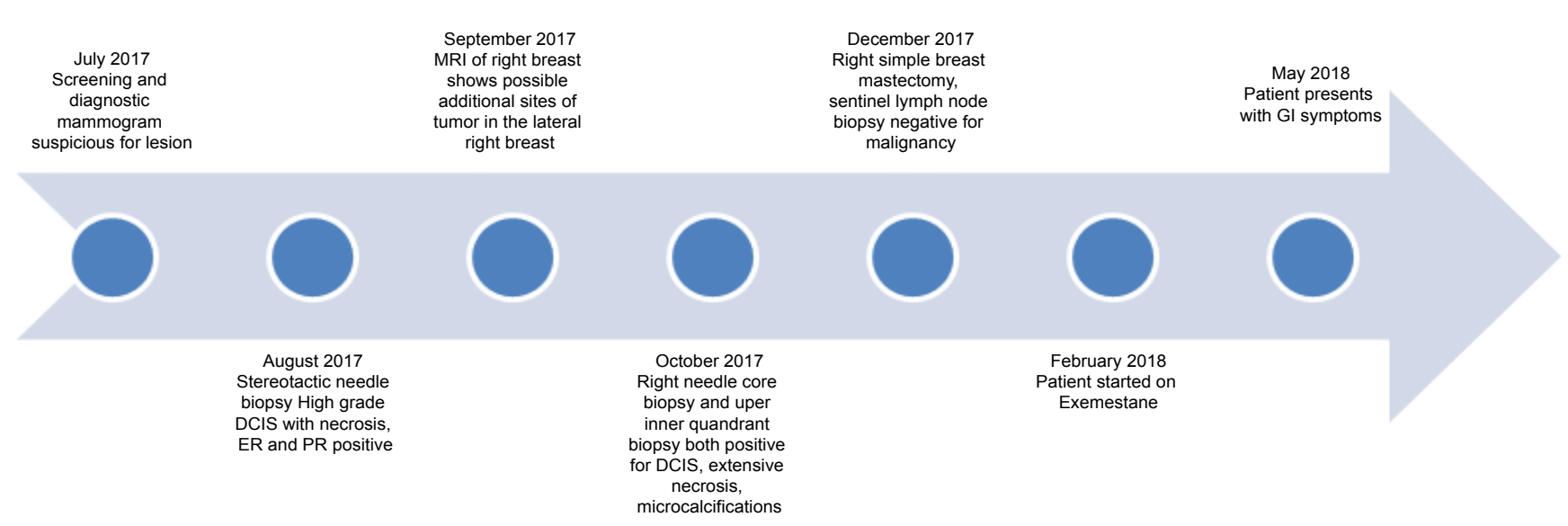

Figure 1: A timeline of events.

suplastat, and biological agents [27-31]. Steroids are the mainstay of treatmentand are thought to be beneficial in EC due to inhibition of eosinophil specific growth factors such as IL-3 and IL-5 [27-31]. Steroid regimens most commonly used in EC are those that mimic regimens used in inflammatory bowel disease [27-31]. Montelukast is an alternative treatment to steroids and works by inhibition of the leukotriene receptor and preventing the recruitment and chemoattraction of eosinophils to the gastrointestinal system [32]. Another alternative option to steroids is the antihistamine ketotifen which stabilizes mast cells and prevents the release of histamine [33]. Lastly, biological agents represent a more novel approach to treating EC and act by using monoclonal antibodies against specific inflammatory mediators [27-31]. Treatment can take up to 2 to 6 weeks. In many cases, patients will not have a recurrence after initial treatment, however, recurrence is still possible and may require alternative long-term treatment options [27-31].

\section{Case Presentation}

The patient is a 60-year-old female with a past medical history positive for hypothyroidism, cataracts, polycystic ovarian syndrome, and glaucoma, who presented to Banner MD Anderson Cancer Center with a new diagnosis of ductal cell carcinoma insitu (DCIS) of the right breast.

Her medical history is also significant for a prior diagnosis of microscopic colitis that was diagnosed on colonoscopy when she was around 40-years-old, when she presented with watery diarrhea. Her main symptom had been diarrhea and she was maintained on pancrelipase and budesonide for the past 20 years without any flares requiring hospitalization or any other medical intervention. Her social history was unremarkable and family history was positive for dementia, hypertension, and father with leukemia as well as prostate cancer at an older age.

Her cancer history dates back to July of 2017 in which the patient presented with an abnormal screen- ing mammogram showing breast imaging-reporting and data system (BIRADS) score of 0 which is indicative of the need for further imaging. A diagnostic mammogram was performed that same month and showed pleiomorphic calcifications, and a stereotactic needle biopsy was recommended. Right breast needle core biopsy and upper inner quadrant biopsy both revealed high grade DCIS with extensive necrosis that was estrogen and progesterone receptor positive. She then underwent a right simple mastectomy and sentinel lymph node biopsy in December of 2017. The sentinel lymph node biopsy was found to be negative for metastatic disease. A timeline of events is outlined below in Figure 1.

We met with the patient in the High-Risk Breast Cancer Clinic on January 26, 2018 and she was evaluated for the need for chemoprevention. Given her high-risk lifetime breast cancer occurrence in the contralateral breast as well as the fact that she was post-menopausal, and her DCIS was strongly estrogen receptor positive, she was determined to be a candidate for exemestane. The patient was started on exemestane in February of 2018.

After nearly 4 months of therapy, in May of 2018, she presented to the emergency room with severe abdominal pain, nausea, vomiting, and diarrhea. She also complained of mucous and a small amount of blood in her stools. The patient had been taking overthe-counter bismuth subsalicylate due to her history of microscopic colitis, which she had discontinued 4 days prior to presentation. A CT of the abdomen and pelvis showed severe colitis of the transverse colon with milder degrees of colitis involving the remaining colonic segments (Figure 2). Laboratory analyses showed mild leukocytosis and normal serum eosinophil count. Stool cultures were negative for Clostridium difficile infection. The patient was admitted for acute on chronic severe colitis and stayed in the hospital for two days. She was started on antibiotic therapy with metronidazole and levofloxacin and was discharged home. No other medications were recently started except for exemestane that would have contributed to 


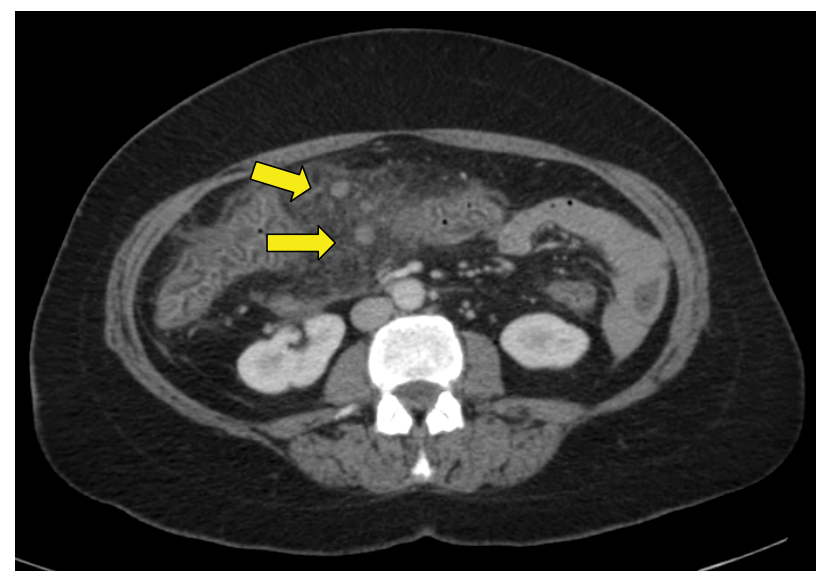

A

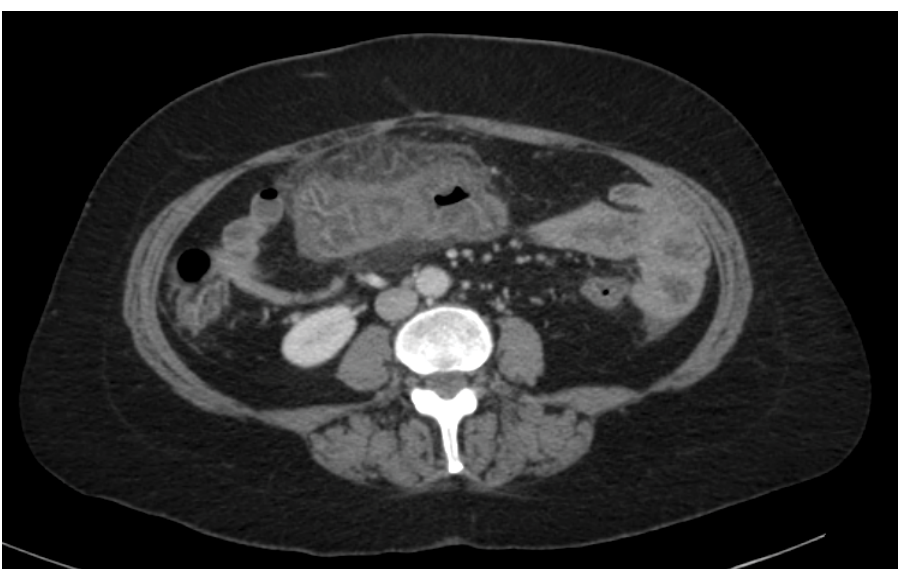

B

Figure 2: a) Inflammation of colon and arrows pointing to enlarged lymph nodes; b) Most severe area of colitis.

the patient's symptoms. She completed gastrointestinal workup as an outpatient.

Outpatient workup included stool cultures, which were negative for Salmonella, Shigella, Aeromonas, Plesiomonas, Escherichia coli, Campylobacter jejuni, and Campylobacter coli, as well as negative for Shiga toxins 1 and 2. Laboratory analysis was significant for elevated lgA levels to $138 \mathrm{mg} / \mathrm{dL}$. An esophagogastroduodenoscopy and colonoscopy were performed and were unrevealing. However, random colon biopsies showed moderate to severe chronic colitis with increased eosinophilia with features suggestive of eosinophilic colitis. The biopsy of the transverse colon ulceration also revealed moderate to borderline severe chronic colitis with increased eosinophilia. The patient was started on corticosteroids and went on a drug holiday with exemestane. Her symptoms resolved in two weeks. Approximately two months later, the patient was started on tamoxifen for breast cancer prevention, which she tolerated well.

\section{Discussion}

We report an interesting case of a 60-year-old lady presenting with an acute episode of eosinophilic colitis 3 months after initiation of an aromatase inhibitor.

Eosinophilic colitis (EC) was first described in 1936 but did not surface in the Western literature until 1959. It is hard to quantify the true prevalence of EC since eosinophilic infiltration of the colon can have other causes including IgE-mediated food allergy, gastroesophageal reflux disease (GERD), and inflammatory bowel disease.

Drug induced EC is an even a rarer entity with only a few case reports described in the literature to date. Some of these case reports have been linked to betalactam antibiotics commonly used in treating infections, while others are related to tacrolimus, carbamazepine, clozapine, rifampin, and non-steroidal anti-inflammatory agents [15-24]. Diagnosis is usually of exclusion and is made from the presence of gastrointestinal symptoms, endoscopic and histological findings as well as the presence of peripheral eosinophilia. Our patient presented with the symptoms as well as the histological findings, but had no peripheral eosinophilia, which can happen in up to $70 \%$ of the cases. The timeline with the inciting drug as well as the quick improvement after stopping exemestane in the absence of other causative agents speaks to a potential exemestane induced EC.

There have been reports on the possible association of aromatase inhibitors with gastrointestinal symptoms, mainly nausea, sickness to the stomach, and diarrhea. Literature search revealed aromatase inhibitors can induce diarrhea in $4-5 \%$ of patients. However, to the best of our knowledge there has only been only one prior case report with an aromatase inhibitor-induced EC from letrozole. The above presented case is actually the first case report with exemestane induced EC.

\section{Conclusion}

In conclusion, attention to the possibility of colitis is required when a patient is on a new medication in the setting of worsening or new onset watery diarrhea. It is important to report these cases and closely monitor those who develop gastrointestinal symptoms while on aromatase inhibitors since these drugs are widely used and are the mainstay of therapy in patients with estrogen receptor positive breast cancer.

\section{Disclosure Statement}

The authors of the case report have no conflicts of interest to disclose.

\section{References}

1. Aromasin (Exemestane) prescribing information (1999) New York: Pfizer.

2. Morden JP, Alvarez I, Bertelli G, Coates AS, Coleman $\mathrm{R}$, et al. (2017) Long-Term Follow-Up of the Intergroup Exemestane Study. J Clin Oncol 35: 2507-2514.

3. Burstein HJ, Prestrud AA, Seidenfeld J, Anderson $H$, Buchholz TA, et al. (2010) American society of clinical oncology clinical practice guidelines update on adjuvant endocrine therapy for women with hormone receptorpositive breast cancer. J Clin Oncol 6: 243-248. 
4. Burstein $\mathrm{HJ}$, Lacchetti $\mathrm{C}$, Anderson $\mathrm{H}$, Buchholz TA, Davidson NE, et al. (2016) Adjuvant endocrine therapy for women with hormone-receptor positive breast cancer: American Society of clinical oncology practice guideline update on ovarian suppression summary. J Clin Oncol 12 390-394.

5. Goss PE, Ingle JN, Alés-Martínez JE, Cheung AM, Chlebowski RT, et al. (2011) Exemestane for BreastCancer Prevention in Postmenopausal Women. N Engl J Med 364: 2381-2391.

6. Visvanathan K, Hurley P, Bantug E, Brown P, Col NF, et al. (2013) Use of Pharmacologic Interventions for Breast Cancer Risk Reduction: American Society of Clinical Oncology Clinical Practice Guideline. J Clin Oncol 31: 2942-2962.

7. De Placido S, Gallo C, De Laurentiis M, Bisagni G, Arpino $G$, et al. (2018) Adjuvant anastrozole versus exemestane versus letrozole, upfront or after 2 years of tamoxifen, in endocrine-sensitive breast cancer (FATA-GIM3): a randomized, phase 3 trial. Lancet Oncology 19: 474-485.

8. Derks MGM, BlokEJ, Seynaeve C, Nortier JWR, Kranenbarg $E M$, et al. (2017) Adjuvant tamoxifen and exemestane in women with postmenopausal early breast cancer (TEAM) 10-year follow-up of a multicenter, open-label, randomized, phase 3 trial. Lancet Oncology 18: 1211-1220.

9. Pagani O, Regan MM, Walley BA, Fleming GF, Colleoni $M$, et al. (2014) Adjuvant exemestane with Ovarian Suppression in Premenopausal Breast Cancer. N Engl J Med 371: 107-118.

10. Campos SM, Guastalla JP, Subar M, Abreu P, Winer EP, et al. (2009) A Comparative Study of Exemestane Versus Anastrozole in Patients with Postmenopausal Breast Cancer with Visceral Metastases. Clin Breast Cancer 9: 39-44.

11. Beresford M, Tumur I, Chakrabarti J, Barden J, Rao N, et al. (2011) A Qualitative Systematic Review of the Evidence Base for Non-cross-resistance between Steroidal and Nonsteroidal Aromatase Inhibitors in Metastatic Breast Cancer. Clin Oncology 23: 209-215.

12. Baselga J, Campone M, Piccart M, Burris HA 3rd, Rugo HS, et al. (2012) Everolimus in Postmenopausal HormoneReceptor-Positive Advanced Breast Cancer. N Engl J Med 366: 520-529.

13. Goss PE, Ingle JN, Pritchard KI, Ellis MJ, Sledge GW, et al. (2013) Exemestane Versus Anastrozole in Postmenopausal Women with Early Breast Cancer: NCIC CTG MA.27-A Randomized Controlled Phase III Trial. J Clin Oncol 31: 1398-1404.

14. Perez EA (2007) Safety profiles of tamoxifen and the aromatase inhibitors in adjuvant therapy of hormoneresponsive early breast cancer. Annals of Oncology 18.

15. McCloskey EV, Hannon RA, Lakner G, Fraser WD, Clack G, et al. (2007) Effects of third generation aromatase inhibitors on bone health and other safety parameters: results of an open, randomized, multi-centre study of letrozole, exemestane, and anastrozole in healthy postmenopausal women. Eur J Cancer 43: 2523-2531.

16. Nicolás-Sánchez FJ, Garreta-Messegue J, Gallel-Vicente P, Gort-Oromí A, Soler-Rosell T, et al. (2011) Colitis eosinofilica asociada a letrozol. J Gastrohep 10: 129-130.

17. Jensen ET, Martin CF, Kappelman MD, Dellon ES (2016) Prevalence of eosinophilic gastritis, gastroenteritis, and colitis: estimates from a national administrative database. J Pediatr Gastroenterol Nutr 62: 36-42.
18. Saeed SA, Integlia MJ, Pleskow RG, Calenda KA, Rohrer RJ, et al. (2006) Tacrolimus-associated eosinophilic gastroenterocolitis in pediatric liver transplant recipients: role of potential food allergies in pathogenesis. Pediatr Transplant 10: 730-735.

19. Karmacharya R, Mino M, Pirl WF (2005) Clozapine-induced eosinophilic colitis. Am J Psychiatry 162: 1386-1387.

20. de Raad AW, Siegersma NC, Clahsen PC, Hagestein-de Bruijn C (2011) [Eosinophilic colitis caused by clozapine]. Ned Tijdschr Geneeskd 155: A3620.

21. Friedberg JW, Frankenburg FR, Burk J, Johnson W (1995) Clozapine-caused eosinophilic colitis. Ann Clin Psychiatry 7: 97-98.

22. Anttila VJ, Valtonen M (1992) Carbamazepine-induced eosinophilic colitis. Epilepsia 33: 119-121.

23. Tajima A, Mine T, Ogata E (1992) Rifampicin-associated ulcerative colitis. Ann Intern Med 116: 778-779..

24. Martin DM, Goldman JA, Gilliam J, Nasrallah SM (1981) Gold-induced eosinophilic enterocolitis: response to oral cromolyn sodium. Gastroenterology 80: 1567-1570.

25. Bridges AJ, Marshall JB, Diaz-Arias AA (1990) Acute eosinophilic colitis and hypersensitivity reaction associated with naproxen therapy. Am J Med 89: 526-527.

26. Jiménez-Sáenz M, González-Cámpora R, Linares-Santiago E, Herrerías-Gutiérrez JM (2006) Bleeding colonic ulcer and eosinophilic colitis: a rare complication of nonsteroidal anti-inflammatory drugs. J Clin Gastroenterol 40: 84-85.

27. Uppal V, Kreiger P, Kutsch E (2016) Eosinophilic gastroenteritis and colitis: a comprehensive review. Clinic Rev Allerg Immunol 50: 175-188.

28. Alfadda AA, Storr MA, Shaffer EA (2011) Eosinophilic colitis: an update on pathophysiology and treatment. Be Med Bull 100: 59-72.

29. Okpara N, Aswad B, Baffy G (2009) Eosinophilic colitis. World J Gastroenterol 15: 2975-2979.

30. Gonsalves N (2007) Food Allergies and Eosinophilic Gastrointestinal Illness. Gastroenterol Clin N AM 36: 75-91.

31. Chen MJ, Chu CH, Lin SC, Shih SC, Wang TE (2003) Eosinophilic gastroenteritis: Clinical experience with 15 patients. World J Gastroenterol 9: 2813-2816.

32. Friesen CA, Kearns GL, Andre L, Neustrom M, Roberts CC, et al. (2004) Clinical Efficacy and Pharmacokinetics of Montelukast in Dyspeptic Children with Duodenal Eosinophilia. J Ped Gastroenterol Nutrition 38: 343-351.

33. Pérez-Millán A, Martín-Lorente JL, López-Morante A, Yuguero L, Sáez-Royuela F (1997) Subserosal Eosinophilic Gastroenteritis Treated Efficaciously with Sodium Cromoglycate. Digestive Diseases and Sciences 42: 342-344. 